\title{
The urodynamic status during psychogenic erection in a patient with a conus medullaris injury. Case report
}

\author{
K Yasuda, ${ }^{1} \mathrm{~T}$ Yamanishi, ${ }^{1} \mathrm{~T}$ Hattori, ${ }^{2} \mathrm{~T}$ Ichikawa, ${ }^{1} \mathrm{H}$ Kitahara ${ }^{3}$ and $\mathrm{J}$ Shimazaki ${ }^{1}$ \\ Department of ${ }^{1}$ Urology, ${ }^{2}$ Neurology and ${ }^{3}$ Orthopedic Surgery, School of Medicine, \\ Chiba University, 1-8-1, Inohana, Chuo-ku, Chiba, Japan.
}

The urodynamic status of a 24 year old male patient with psychogenic erection who sustained a conus medullaris lesion from a burst fracture of the first lumbar vertebra is reported. During the initial measurement the external urinary sphincter pressure began to rise from the base pressure of $35 \mathrm{~cm} \mathrm{H}_{2} \mathrm{O} 5$ seconds after the beginning of audiovisual sexual stimulation and reached the peak of $110 \mathrm{~cm} \mathrm{H}_{2} \mathrm{O}$. In parallel, the bladder neck pressure gradually rose from its base pressure of $5 \mathrm{~cm} \mathrm{H}_{2} \mathrm{O}$ to a maximal pressure of $115 \mathrm{~cm} \mathrm{H}_{2} \mathrm{O}$ and then ejaculation occurred. The difference is no more than $5 \mathrm{~cm} \mathrm{H}_{2} \mathrm{O}$ and this small difference in pressure inhibits retrograde ejaculation.

Keywords: urodynamic status; psychogenic erection; Rigiscan; conus medullaris injury.

\section{Introduction}

The urodynamic status can be easily appreciated from animal data during erection and ejaculation. ${ }^{1,2}$ Seminal emission from the prostate and the seminal vesicles produces a bolus of semen in the prostatic urethra, and the external urinary sphincter must close during seminal emission. Then, just before ejaculation occurs, the external urinary sphincter relaxes, and the bladder neck closes during ejaculation. ${ }^{1,2}$ There have been, however, few human studies to the best of our knowledge, probably because of technical difficulties, especially during ejaculation.

The purpose of this study is to examine the urodynamic status of the lower urinary tract during erection and ejaculation as well as the effects of drug therapy in a patient with psychogenic erection, who is able to ejaculate in the presence of other people.

\section{Materials and methods}

On 11 May 1987, a 24 year old male sustained a burst fracture of the first lumbar vertebra. Six days later, L1 vertebrectomy and an anterior intervertebral fusion were performed. By July, he could manage to void with straining. In August, he had a balanced bladder with a small amount of residual urine $(10-50 \mathrm{ml})$ and had slight incontinence. At the beginning of 1988, whilst reading a pornographic book, he became sexually aroused, but with only incomplete erection and ejaculated within a minute of arousal. He wanted the penis to become more tumescent, to increase in rigidity and to have an increase of time from erection to ejaculation during sexual intercourse. He had no complaints about sexual capabilities before his spinal injury.

A cystogram revealed the bladder neck to be slightly open at rest. Also, a 1 hour pad test demonstrated a urinary incontinence of $6 \mathrm{gm}$. Neurological examination showed absent pinprick and cotton-wool touch sensations around the perianal area. Anal tone was decreased and the bulbocavernosus reflex was sluggish. Motor power, sensation and the deep reflexes in the lower extremities were normal. Achilles tendon reflexes and patellar tendon reflexes were normal.

\section{Measurements before drug therapy (initial measurements) (Fig 1)}

Urodynamic study: A 5-micro-transducer catheter was inserted into the lower urinary 


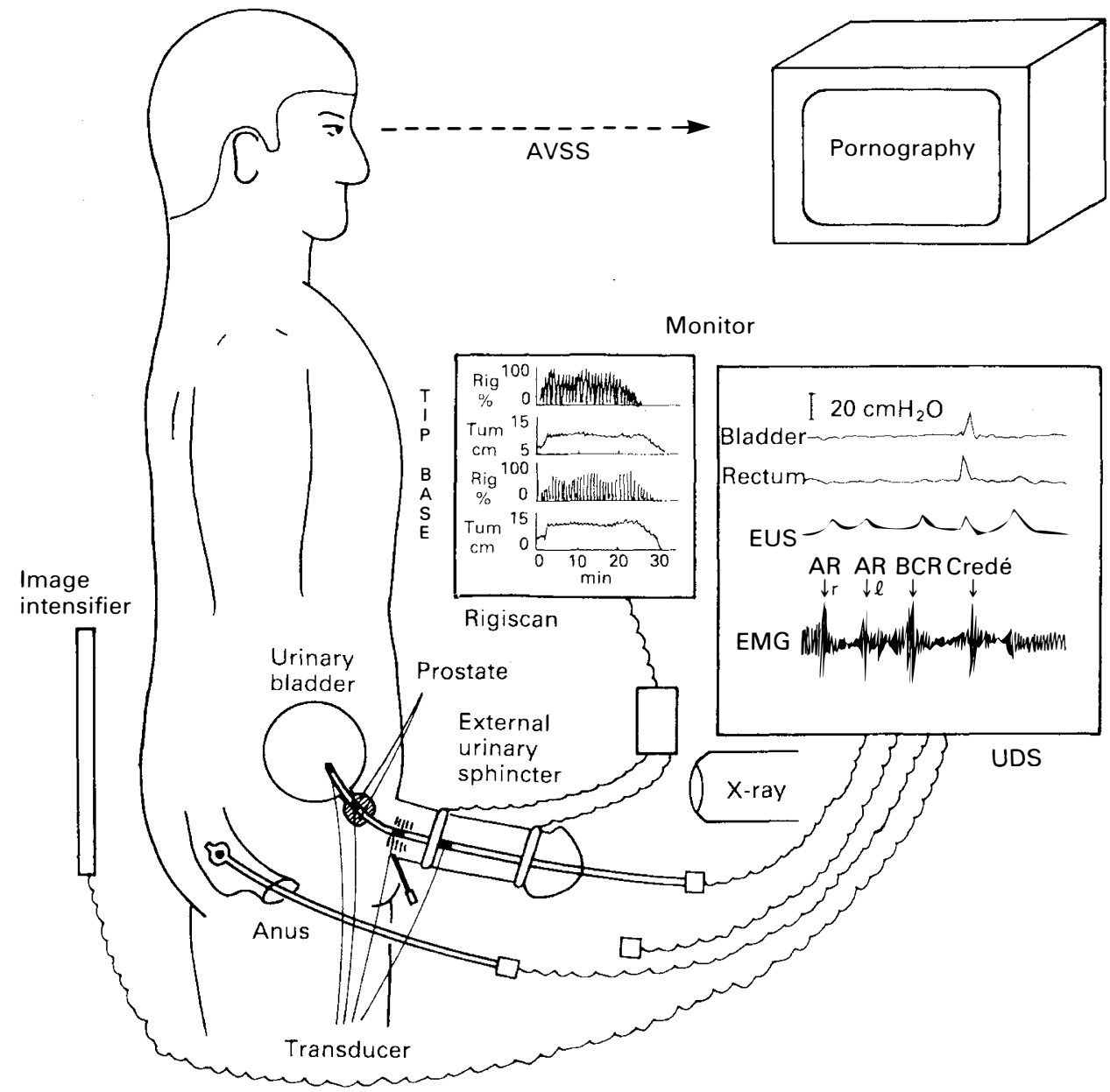

Figure 1 Audiovisual arrangement.

tract. A $20 \%$ dye solution $(350 \mathrm{ml})$ was instilled into the bladder to confirm the position of the transducer under X-ray image. The bladder pressure, bladder neck pressure, external urinary sphincter pressure and bulbous urethral pressure were measured by the method of Rossier \& Fam. ${ }^{3,4}$ The rectal pressure was measured by the use of a monomicrotip-transducer catheter. The external urinary sphincter EMG was recorded with needle electrodes.

Rigiscan: The penile circumference and rigidity at the penile base and tip were measured by Rigiscan ${ }^{\circledR}$ (Fig 1) during audiovisual sexual stimulation (AVSS). ${ }^{5}$
Measurements of penile circumference and rigidity under drug therapy

Rigiscan: The penile circumference and rigidity were measured in the same way as had been done before drug therapy on the 7th day of a 14-day treatment with yohimbine with a daily dose of $12 \mathrm{mg}$, t.i.d., and on the 7th day of a 14-day treatment with guanethidine in a daily dose of $20 \mathrm{mg}$, b.i.d.

\section{Results}

In the initial measurement, the external urinary sphincter pressure began to rise from the base pressure of $35 \mathrm{~cm} \mathrm{H}_{2} \mathrm{O}$ 
5 seconds after the beginning of AVSS and reached the peak of $110 \mathrm{~cm} \mathrm{H}_{2} \mathrm{O}\left(\mathrm{T}_{\text {max }}\right.$ : approx $40 \mathrm{~s})$. In parallel, the bladder neck pressure gradually rose from its base pressure of $5 \mathrm{~cm} \mathrm{H} \mathrm{H}_{2} \mathrm{O}$ to a maximal pressure of $115 \mathrm{~cm} \mathrm{H}_{2} \mathrm{O}$ at which pressure ejaculation occurred. After 30 seconds from the beginning of AVSS, the bladder pressure rose from its base line of $5 \mathrm{~cm} \mathrm{H}_{2} \mathrm{O}$ to a maximum of $40 \mathrm{~cm} \mathrm{H}_{2} \mathrm{O}$, while the rectal pressure did not change (Fig 2).

\section{Effect of drug therapy}

The maximal circumference of the penile base was $9.5 \mathrm{~cm}$ initially, $10 \mathrm{~cm}$ by yohimbine and $11 \mathrm{~cm}$ by guanethidine. The maximal circumference of the penile tip was $10 \mathrm{~cm}$ initially, $8.5 \mathrm{~cm}$ by yohimbine and $9.5 \mathrm{~cm}$ by guanethidine. The rigidity rate, calculated as a ratio of rigidity under AVSS to that at rest (without AVSS), increased by $20 \%$ initially, $85 \%$ by yohimbine and $90 \%$ by guanethidine at the penile base and by $30 \%$ initially, $75 \%$ by yohimbine and $100 \%$ by guanethidine at the penile tip. The time interval from the start of AVSS to ejaculation was 40 seconds initially, 2 minutes by yohimbine and 3 minutes by guanethidine (Fig 3). The semen volume of each ejaculation ranged from 1.5 to $2.0 \mathrm{ml}$. No retrograde ejaculation into the bladder was observed.

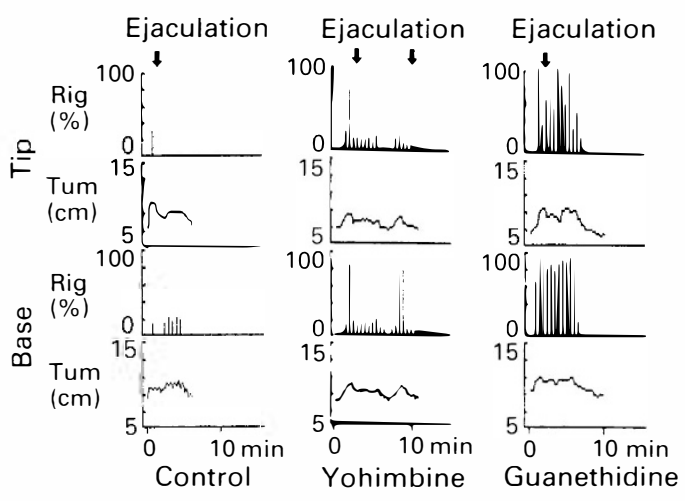

Figure 3 Effects of yohimbine and of guanethidine on ejaculation.

\section{Discussion}

There have been no detailed reports on the urodynamic status from the beginning of sexual arousal to ejaculation. From the results of this study, detrusor contraction occurred during erection and at ejaculation. This may not be a common finding but would appear to explain the reports of urinary incontinence that occur during sexual intercourse with females. The endoscopic film has shown that the coliculus moves downward at ejaculation, suggesting relaxation of the pelvic floor muscles. However, as was found in this study, the

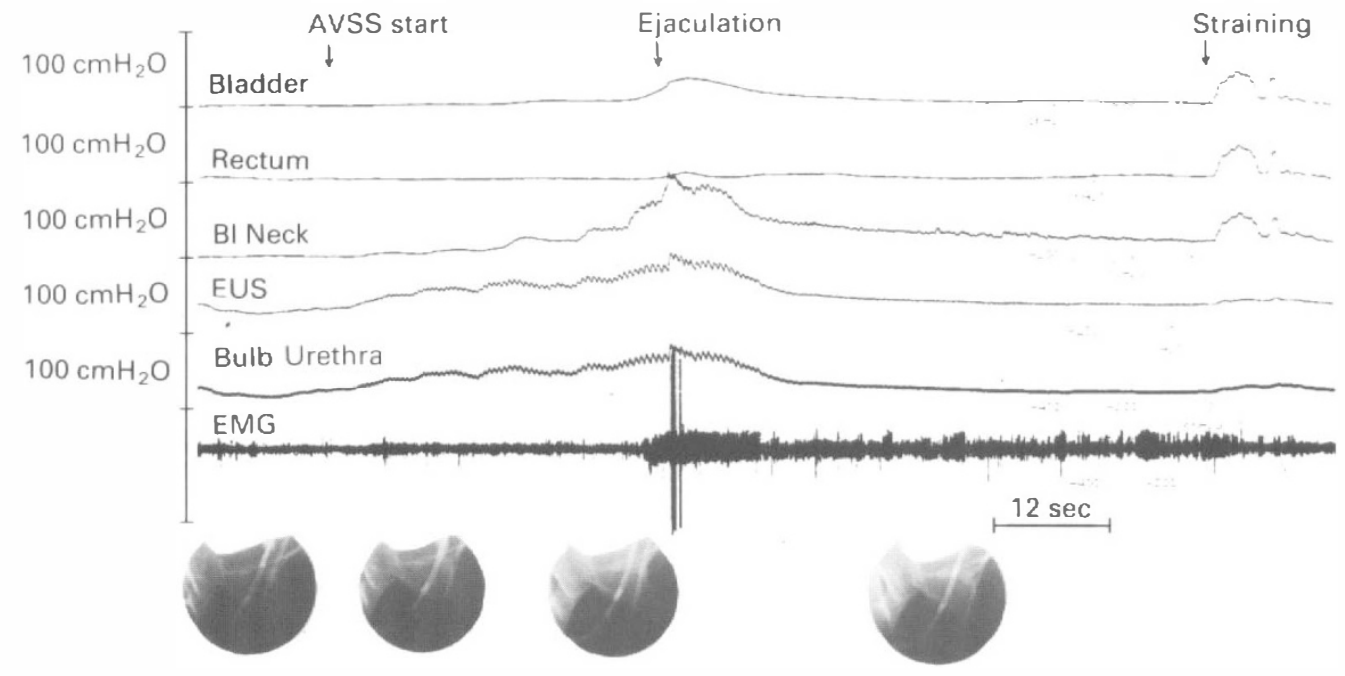

Figure 2 Bladder, urethral and rectal pressures. 
pressure of the external urinary sphincter continuously increased over the baseline during erection until there was ejaculation, which means that this muscle did not relax at ejaculation. During ejaculation, the bladder neck pressure was higher than the external urinary sphincter pressure. The difference was no more than $5 \mathrm{~cm} \mathrm{H}_{2} \mathrm{O}$ and it is interesting that this small difference in pressure inhibited retrograde ejaculation. A cystogram of our patient showed the bladder neck to be slightly open, but it closed during erection and ejaculation (Fig 2). Consequently, the bladder neck opening found on the cystogram was not always a cause of retrograde ejaculation.

For penile rigidity, tumescence and time interval to ejaculation, guanethidine seemed to be more effective than yohimbine at least by our dosing schedule. Yohimbine has only a pure alpha-2 blocking effect while guanethidine has alpha-1 and alpha- 2 blocking effects. The alpha-1 blocking effect of guanethidine may induce retrograde ejaculation and delay the time interval to ejaculation, ${ }^{6}$ although no retrograde ejaculation was observed during the treatment with guanethidine in our patient.

\section{References}

1 Kimura Y, Miyata K, Adachi K, Kisaki N (1975) Peripheral nerves controlling the closure of internal urethral orifice during ejaculation. Urol Int 30: 218-227.

2 Kihara K, Sato K, Ando M, Sato T, Oshima H (1991) Lumbosacral sympathetic trunk as a compensatory pathway for seminal emission after bilateral hypogastric nerve transections in the dog. J Urol 145: 640-643.

3 Rossier AB, Fam BA (1986) Microtransducer catheter in evaluation of neurogenic bladder function. Urology 27: $371-378$.

4 Yasuda K, Nagashima K, Murayama N, Yamanishi T, Tojo M, Shimazaki J (1991) Change of external urethral sphincter function in prostatic patients. Urol Int 47 (suppl 1): 43-47.

5 Kaneko S, Bradly WE (1986) Evaluation of erectile dysfunction with continuous monitoring of penile rigidity. J Urol 136: 1026-1029.

6 Nelson RP (1988) Nonoperative management of impotence. J Urol 139: 2-5. 